University of Nebraska - Lincoln

DigitalCommons@University of Nebraska - Lincoln

\title{
Magnetic properties of nickel hydroxide nanoparticles
}

X. H. Liu

Shenyang National Laboratory for Materials Science, Institute of Metal Research and International Center for Materials Physics, Chinese Academy of Sciences, Shenyang 110016, People's Republic of China

W. Liu

Shenyang National Laboratory for Materials Science, Institute of Metal Research and International Center for Materials Physics, Chinese Academy of Sciences, Shenyang 110016, People's Republic of China

X. K. LV

Shenyang National Laboratory for Materials Science, Institute of Metal Research and International Center for Materials Physics, Chinese Academy of Sciences, Shenyang 110016, People's Republic of China

F. Yang

Shenyang National Laboratory for Materials Science, Institute of Metal Research and International Center for Materials Physics, Chinese Academy of Sciences, Shenyang 110016, People's Republic of China

X. Wei

University of Nebraska-Lincoln

See next page for additional authors

Follow this and additional works at: https://digitalcommons.unl.edu/physicssellmyer

Part of the Physics Commons

Liu, X. H.; Liu, W.; Lv, X. K.; Yang, F.; Wei, X.; Zhang, Z. D.; and Sellmyer, David J., "Magnetic properties of nickel hydroxide nanoparticles" (2010). David Sellmyer Publications. 223.

https://digitalcommons.unl.edu/physicssellmyer/223

This Article is brought to you for free and open access by the Research Papers in Physics and Astronomy at DigitalCommons@University of Nebraska - Lincoln. It has been accepted for inclusion in David Sellmyer Publications by an authorized administrator of DigitalCommons@University of Nebraska - Lincoln. 


\section{Authors}

X. H. Liu, W. Liu, X. K. Lv, F. Yang, X. Wei, Z. D. Zhang, and David J. Sellmyer 


\title{
Magnetic properties of nickel hydroxide nanoparticles
}

\author{
X. H. Liu, ${ }^{1}$ W. Liu, ${ }^{1,2, a)}$ X. K. Lv, ${ }^{1}$ F. Yang,${ }^{1}$ X. Wei ${ }^{2}$ Z. D. Zhang, ${ }^{1}$ and D. J. Sellmyer ${ }^{2}$ \\ ${ }^{1}$ Shenyang National Laboratory for Materials Science, Institute of Metal Research and International Center \\ for Materials Physics, Chinese Academy of Sciences, Shenyang 110016, People's Republic of China \\ ${ }^{2}$ Department of Physics and Astronomy and Nebraska Center for Materials and Nanoscience, \\ University of Nebraska-Lincoln, Lincoln, Nebraska 68588, USA
}

(Received 1 March 2010; accepted 3 March 2010; published online 29 April 2010)

\begin{abstract}
The magnetic properties of $10 \mathrm{~nm}$ size $\mathrm{Ni}(\mathrm{OH})_{2}$ nanoparticles prepared by sol-gel method have been studied. The magnetic moments increase with decreasing temperature in a low applied field, which is due to the spin-frozen-like state at low temperatures, and the metamagnetic transition is not clearly observed even in an applied field of $70 \mathrm{kOe}$ due to the size effect. Furthermore, the transition from paramagnetic to antiferromagnetic in the $\mathrm{Ni}(\mathrm{OH})_{2}$ nanoparticles occurs at lower temperature (22 K). (C) 2010 American Institute of Physics. [doi:10.1063/1.3374468]
\end{abstract}

\section{INTRODUCTION}

Magnetic nanoparticles are a subject of intense research due to their unique magnetic properties which make them very appealing from both the theoretical and technological point of view. ${ }^{1-11}$ The exchange-bias phenomenon, i.e., the hysteresis loop shifts in the applied field, was first discovered by Meiklejohn and Bean in oxide-coated Co particles. ${ }^{12} \mathrm{Re}$ cently, the research on the exchange bias has been attractive, because of its fundamentally important role in spin valve and tunneling devices. ${ }^{13,14}$ The antiferromagnetic (AFM) NiO nanoparticles have been well studied due to their potential for exhibiting magnetization reversal by the quantum tunneling as well as for their technological application. ${ }^{15}$ The magnetic properties of $\mathrm{Ni}(\mathrm{OH})_{2}$ nanoparticles have attracted little attention, and one of the important application of $\mathrm{Ni}(\mathrm{OH})_{2}$ is in rechargeable battery system, ${ }^{16}$ where its structural and compositional characteristics greatly influence the properties during reversible in recharging. ${ }^{17}$ Recently, Tiwari and Rajeev ${ }^{18}$ reported that the $\mathrm{Ni}(\mathrm{OH})_{2}$ nanoparticles prepared by the sol-gel exhibited a paramagnetic (PM)-toferromagnetic (FM) transition at low temperatures, and found superparamagnetic blocking at lower temperature. However, Takada et al. ${ }^{19}$ reported that bulk $\mathrm{Ni}(\mathrm{OH})_{2}$ was an antiferromagnet exhibiting a metamagnetic property with the Neél temperature of $30 \mathrm{~K}$, and a field-induced transition took place when the applied field was larger than the critical field. In this paper, we report magnetic properties of $\mathrm{Ni}(\mathrm{OH})_{2}$, and find that the transition in the $\mathrm{Ni}(\mathrm{OH})_{2}$ nanoparticles is from paramagnetic to antiferromagnetic at low temperature, which is similar to bulk properties. Furthermore, the exchangebias phenomenon and the coercivity field with the variation in temperature are investigated.

\section{EXPERIMENT}

$\mathrm{Ni}(\mathrm{OH})_{2}$ nanoparticles were fabricated by a sol-gel method, by reacting aqueous solutions of nickel sulfate $\mathrm{NiSO}_{4} \cdot 6 \mathrm{H}_{2} \mathrm{O}$ and sodium hydroxide $\mathrm{NaOH}$ at $p \mathrm{H}=12$ at room temperature with continuously stirring. The obtained

${ }^{\text {a)} E l e c t r o n i c ~ m a i l: ~ w l i u @ i m r . a c . c n . ~}$ green sol of $\mathrm{Ni}(\mathrm{OH})_{2}$ was centrifuged to obtain a gel of $\mathrm{Ni}(\mathrm{OH})_{2}$. The resulting gel was washed several times with distilled water until free of sulfate ions. The gel was then dried at $353 \mathrm{~K}$ for several hours to obtain green-colored $\mathrm{Ni}(\mathrm{OH})_{2}$ powders. X-ray diffraction (XRD) analysis was conducted using $\mathrm{Cu} K_{\alpha}$ radiation. The surface of the powder was characterized by X-ray photoelectron spectroscopy (XPS), and all the XPS measurements were carried out with reference to $\mathrm{C} 1 s$ binding energy $(\mathrm{BE})(284.5 \mathrm{eV})$ as internal standard. The magnetic properties were measured by a superconducting quantum interface device from 5 to $200 \mathrm{~K}$.

\section{RESULTS AND DISCUSSION}

The XPS spectra of $\mathrm{Ni} 2 p^{3 / 2}$ and $\mathrm{Ni} 2 p^{1 / 2}$ energy levels are shown in Fig. 1. It is seen that the BE values of $\mathrm{Ni} 2 p^{3 / 2}$ are 855.6 and $861.5 \mathrm{eV}$, corresponding to the $\mathrm{Ni}(\mathrm{OH})_{2}$ and $\mathrm{NiO}$, respectively. Accordingly, the values of $\mathrm{Ni} 2 p^{1 / 2}$ are 873.3 and $880.8 \mathrm{eV}$, corresponding to these two contributions. Based on the analysis above, it is confirmed that $\mathrm{NiO}$ exists on the surface of the powders though its content may be quite small. The XRD pattern in the inset of Fig. 1 confirms the single-phase state of the $\mathrm{Ni}(\mathrm{OH})_{2}$ particles. How-

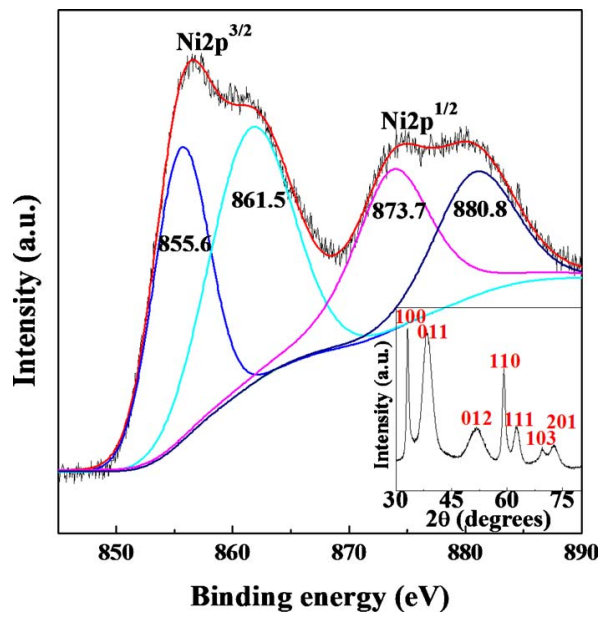

FIG. 1. (Color online) XPS spectra of Ni $2 p^{3 / 2}$ and $\mathrm{Ni} 2 p^{1 / 2}$ of powder. Inset: the XRD pattern of $\mathrm{Ni}(\mathrm{OH})_{2}$. 


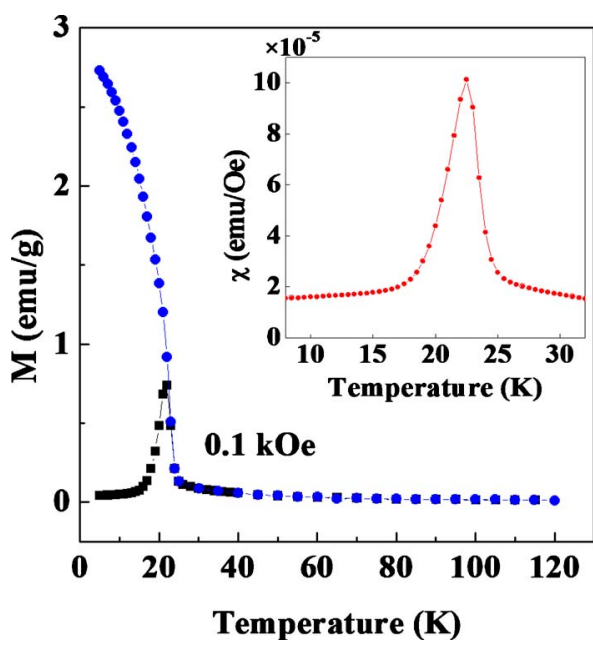

FIG. 2. (Color online) Temperature dependences of ZFC and FC magnetization in applied field of $0.1 \mathrm{kOe}$. Inset: the ac susceptibility vs temperature.

ever, the diverse peaks having very different peak width found in XRD pattern may be due to stacking faults in the material. ${ }^{20}$ As the (100) peak is not affected by stacking fault broadening, the grain size of the sample was determined to be about $10 \mathrm{~nm}$ by the Scherrer formula, ${ }^{21}$ which could approximately represent the average grain size of the sample.

Temperature dependences of zero-field-cooling (ZFC) and field-cooling (FC) magnetization in a magnetic field of 0.1 kOe are shown in Fig. 2. The inset in Fig. 2 presents the ac susceptibility versus temperature. It is clear that the ZFC magnetization increases and reaches a maximum at $22 \mathrm{~K}$, and then decreases with increasing temperature. Furthermore, the $\chi$ versus $T$ curve shows the peak at $22.5 \mathrm{~K}$, which is in agreement with the ZFC curve. In order to investigate the variation in magnetization with applied field, the ZFC-FC curves in applied fields of 1,10 , and $50 \mathrm{kOe}$ are shown in Fig. 3. It can be seen that the bifurcate points of the magnetization curves decrease with increasing applied field. Furthermore, the slopes of the FC curve increase with increasing the applied field below the temperature corresponding to the peak, and the value of slope changes from negative at a small

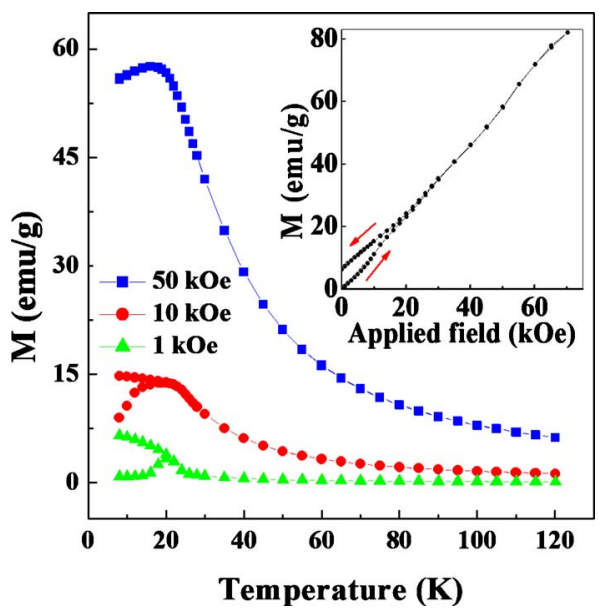

FIG. 3. (Color online) Temperature dependences of ZFC and FC magnetization curves in applied field of 1,10 , and $50 \mathrm{kOe}$. Inset: ZFC $M-H$ curve in applied field of $70 \mathrm{kOe}$ at $10 \mathrm{~K}$. applied field to positive at $50 \mathrm{kOe}$. The inset in Fig. 3 presents the ZFC $M-H$ curve measured from zero to $70 \mathrm{kOe}$ and then back to zero.

From the results above, the peak at around $22 \mathrm{~K}$ would be a freezing temperature of AFM phase. The decrease in the ordered temperature $(22 \mathrm{~K})$ of $\mathrm{Ni}(\mathrm{OH})_{2}$, compared with $30 \mathrm{~K}$ reported in Ref. 19, is due to the decrease in grain size of $\mathrm{Ni}(\mathrm{OH})_{2} \cdot{ }^{14}$ The grain size is about several nanometers in our system, but $2 \sim 5 \mu$ in size in Ref. 19. Moreover, the FC curve in low field in Fig. 2 exhibits ferromagnetic-like property at $25 \mathrm{~K}$, which is quite different from the AFM property in $\mathrm{Co}(\mathrm{OH})_{2}$ nanoparticles. ${ }^{22}$ Figure 3 presents the decrease of FC magnetization with decreasing temperature at $50 \mathrm{kOe}$, indicating that this is a transition from PM to AFM. Furthermore, the spin-frozen-like state occurs at low field, but it will be overcome at a quite large applied field, ${ }^{14,23}$ so that the AFM state gradually increases with increasing applied field, corresponding to the increase in the slope of FC curve as presented in Fig. 3. Moreover, the spin-frozen-like state will occur at low temperatures in low field for FM or AFM nanoparticles. ${ }^{5,7,14}$ Usually, the decrease in the size of materials does not change the nature of the exchange interactions extent on the surface, therefore, the interaction in the $\mathrm{Ni}(\mathrm{OH})_{2}$ nanoparticles is still antiferromagnetic when the surface spin-frozen-like state is overcome, the same as that in the bulk $\mathrm{Ni}(\mathrm{OH})_{2}{ }^{19}$

From the inset in Fig. 3, it is found that not clear metamagnetic transition occurs even up to the applied field of 70 kOe. Therefore, the size effect and the interfacial effect would greatly affect the interaction between spin moments. ${ }^{24}$ Mills $^{25}$ reported that metamagnetic transition may occur when $H$ exceeds the value $H_{\mathrm{cr}}=\left(2 H_{E} H_{A}\right)^{1 / 2}$, at $T=0$, where $H_{E}$ and $H_{A}$ are effective exchange field and anisotropy field, respectively. Similarly, in our system, the $H_{E}$ may be greatly enhanced due to the strong interaction between $\mathrm{NiO}$ and $\mathrm{Ni}(\mathrm{OH})_{2}$, and the $H_{A}$ may be also enhanced in exchange biased systems. ${ }^{14}$ Because large $H_{E}$ and $H_{A}$ lead to higher $H_{\mathrm{cr}}$ than the applied field, the metamagnetic transition is not found clearly in our system (also in Ref. 18).

Furthermore, the exchange bias phenomenon in $\mathrm{Ni}(\mathrm{OH})_{2}$ nanoparticles with the surface of $\mathrm{NiO}$ is studied at low temperatures. The hysteresis loops of $\mathrm{Ni}(\mathrm{OH})_{2}$ at $5,10,15$, and $20 \mathrm{~K}$ in applied fields up to $50 \mathrm{kOe}$, after FC at a cooling field of $5 \mathrm{kOe}$ are given in Fig. 4. The values of exchange bias field $H_{\mathrm{EX}}$ (defined as Ref. 6) and $H_{\mathrm{C}}$ decrease with increasing temperature, which are plotted in Fig. 5. The hysteresis loop at $5 \mathrm{~K}$ in a cooling field of $5 \mathrm{kOe}$ is presented in inset of Fig. 5, in which the $H_{\mathrm{EX}}$ and irreversibility field $\left(H_{\mathrm{ir}}\right)$ are marked by arrows. It is found that the values of $H_{\mathrm{EX}}$ decrease rapidly from 5 to $12 \mathrm{~K}$ and decrease slowly from 12 to $20 \mathrm{~K}$. Accordingly, the value of $H_{\mathrm{C}}$ increases rapidly from 20 to $10 \mathrm{~K}$ and varies slowly with further decreasing temperature. Both the $H_{\mathrm{EX}}$ and $H_{\mathrm{C}}$ nearly disappear at $20 \mathrm{~K}$, around the freezing temperature of $22 \mathrm{~K}$.

Here, we try to understand why the exchange bias phenomenon occurs in the present powders. In magnetic nanoparticles system, the surface spins may be frozen at low temperatures. ${ }^{5-7,26,27}$ Similarly, the exchange interaction between the surface spins and core moments occurs, leading to 


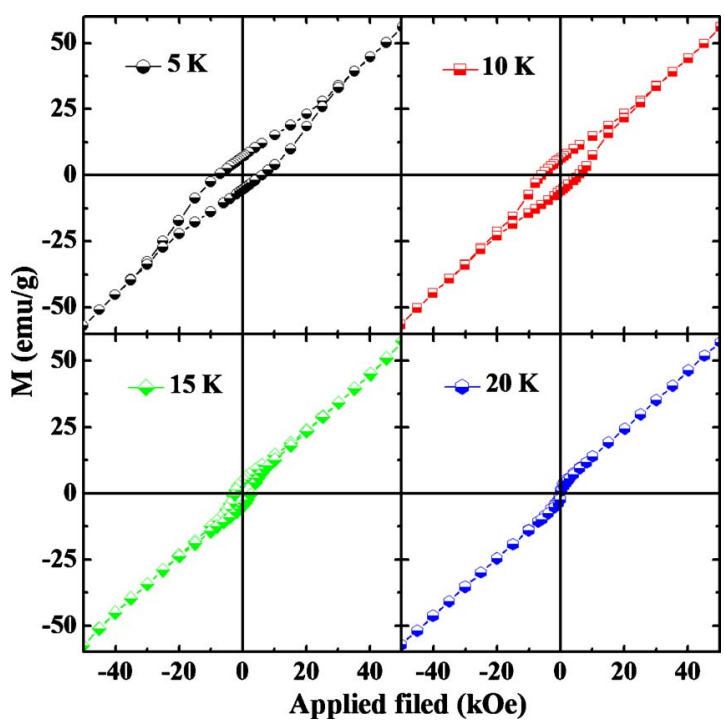

FIG. 4. (Color online) Hysteresis loops at 5, 10, 15, and $20 \mathrm{~K}$ recorded at applied fields up to $50 \mathrm{kOe}$, after $\mathrm{FC}$ at cooling field of $5 \mathrm{kOe}$, respectively.

the exchange bias field in $\mathrm{Ni}(\mathrm{OH})_{2}$ nanoparticles. Moreover, from XPS analysis in Fig. 1, few content of $\mathrm{NiO}$ existed in the surface of the powders with AFM/AFM coupling would take very important role in exchange interaction. ${ }^{28,29}$ Furthermore, in nanoparticles the reduction in the size of AFM systems results in uncompensated moments, which are predominantly at the surface. This uncompensated spins have a FMlike behavior, giving rise to remanence and coercivity in our hysteresis loops (see Fig. 4). ${ }^{5}$ Therefore, the exchange coupling between these uncompensated spins and the AFM core (or $\mathrm{NiO}$ ) would be contained in total energy. All exchange couplings mentioned above will give rise to some magnetic properties: loop shifts and enhancement of coercivity. In addition, the vertical shift is usually found in AFM/FM films and it is very difficult to observe this phenomenon in AFM/ $\mathrm{FM}$, antiferromagnetic/ferrimagnetic (AFM/FI) or AFM/ AFM powder systems, which is not clear in our system. Finally, the interface between two AFM phases would change from compensated to uncompensated as defects introduced

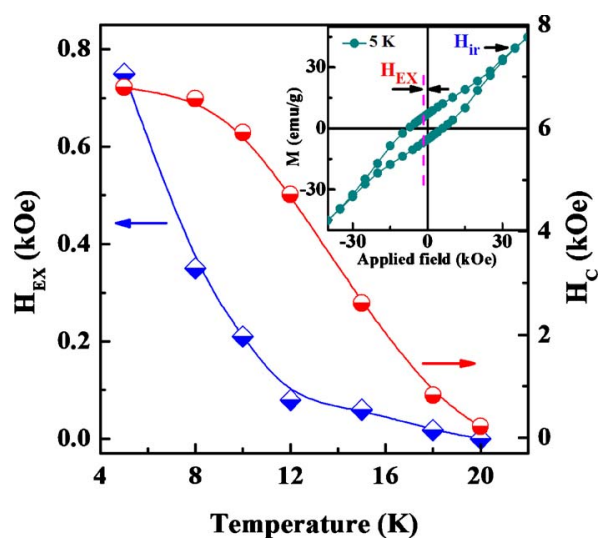

FIG. 5. (Color online) Temperature dependence of $H_{\mathrm{EX}}$ and $H_{\mathrm{C}}$ measured in applied field of $50 \mathrm{kOe}$, after FC at a cooling field of $5 \mathrm{kOe}$. Inset: hysteresis loop at $5 \mathrm{~K}$ in a cooling field of $5 \mathrm{kOe}$, the $H_{\mathrm{EX}}$ and irreversibility field are marked by arrows. into, thus the defect, such as strains and stacking faults, may be also affect the magnetic properties of sample. ${ }^{14}$

\section{CONCLUSION}

It is shown that the transition in the $\mathrm{Ni}(\mathrm{OH})_{2}$ nanoparticles is from PM to AFM, which is similar to bulk. The magnetic moments increase with decreasing temperature in a low applied field, which is due to the spin-frozen-like state at low temperatures because of the small size effect. Not clear observation of the metamagnetic transition is due to the size effect.

\section{ACKNOWLEDGMENTS}

This work has been supported by the National Nature Science Foundation of China under Project Nos. 50831006 and 50971123 and National Basic Research Program (Grant No. 2010CB934603) of China, the Ministry of Science and Technology of China. Research at Nebraska (X.W. and D.J.S) supported by NSF-MRSEC (Grant No. DMR0820521).

\section{ZZ. D. Zhang, J. Mater. Sci. Technol. 23, 1 (2007).}

${ }^{2}$ C. Kittel, Phys. Rev. 70, 965 (1946).

${ }^{3}$ E. M. Chudnovsky and L. Cunther, Phys. Rev. Lett. 60, 661 (1988).

${ }^{4}$ K. Yakushiji, S. Mitani, F. Ernult, K. Takanashi, and H. Fujimori, Phys. Rep. 451, 1 (2007).

${ }^{5}$ R. H. Kodama, A. S. Makhlouf, and A. E. Berkowitz, Phys. Rev. Lett. 79, 1393 (1997).

${ }^{6}$ X. H. Liu, W. B. Cui, X. K. Lv, W. Liu, X. G. Zhao, D. Li, and Z. D. Zhang, J. Appl. Phys. 103, 103906 (2008).

${ }^{7}$ B. Martínez, X. Obradors, L. Balcells, A. Rouanet, and C. Monty, Phys. Rev. Lett. 80, 181 (1998).

${ }^{8}$ S. A. Makhlouf, J. Magn. Magn. Mater. 272-276, 1530 (2004).

${ }^{9}$ X. H. Liu, W. B. Cui, X. K. Lv, W. Liu, X. G. Zhao, D. Li, and Z. D. Zhang, J. Phys. D 41, 105005 (2008).

${ }^{10}$ R. H. Kodama and A. E. Berkowitz, Phys. Rev. B 59, 6321 (1999).

${ }^{11}$ R. H. Kodama, A. E. Berkowitz, E. J. McNiff, Jr., and S. Foner, Phys. Rev. Lett. 77, 394 (1996).

${ }^{12}$ W. H. Meiklejohn and C. P. Bean, Phys. Rev. 102, 1413 (1956).

${ }^{13}$ J. Nogués, D. Lederman, T. J. Moran, and I. K. Schuller, Phys. Rev. Lett. 76, 4624 (1996).

${ }^{14}$ J. Nogués, J. Sort, V. Langlais, V. Skumryev, S. Suriñach, J. S. Muñoz, and M. D. Baró, Phys. Rep. 422, 65 (2005).

${ }^{15}$ P. C. E. Stamp, E. M. Chudnovsky, and B. Barbara, Int. J. Mod. Phys. B 6, 1355 (1992).

${ }^{16}$ K. Watanabe and N. Kumagai, J. Power Sources 76, 167 (1998).

${ }^{17}$ J. T. Richardson and W. O. Milligan, Phys. Rev. 102, 1289 (1956).

${ }^{18}$ S. D. Tiwari and K. P. Rajeev, Phys. Rev. B 77, 224430 (2008).

${ }^{19}$ T. Takada, Y. Bando, M. Kiyama, and H. Miyamoto, J. Phys. Soc. Jpn. 21, 2745 (1966).

${ }^{20}$ M. Casas-Cabanas, J. Power Sources 174, 414 (2007).

${ }^{21}$ H. P. Klug and L. E. Alexander, X-ray Diffraction Procedures for Polycrystalline and Amorphous Materials, 2nd ed. (Wiley, New York, 1974), Chap. 9.

${ }^{22}$ X. H. Liu, W. Liu, W. J. Hu, S. Guo, X. K. Lv, W. B. Cui, X. G. Zhao, and Z. D. Zhang, Appl. Phys. Lett. 93, 202502 (2008).

${ }^{23}$ L. D. Bianco, D. Fiorani, A. M. Testa, E. Bonetti, and L. Signorini, Phys. Rev. B 70, 052401 (2004).

${ }^{24}$ O. Özdemir, D. J. Dunlop, and T. S. Berquó, Geochem., Geophys., Geosyst. 9, Q10Z01 (2008).

${ }^{25}$ D. L. Mills, Phys. Rev. Lett. 20, 18 (1968).

${ }^{26}$ A. H. Morrish and K. Haneda, J. Appl. Phys. 52, 2492 (1981).

${ }^{27}$ D. Lin, A. C. Nunes, C. F. Majkrzak, and A. E. Berkowitz, J. Magn. Magn. Mater. 145, 343 (1995).

${ }^{28}$ S. MØrup, Hyperfine Interact. 144-145, 347 (2002).

${ }^{29}$ C. Frandsen, J. Magn. Magn. Mater. 266, 36 (2003). 